\title{
Conjugated polymer and triphenylamine derivative co-doped nanoparticles for photothermal and photodynamic antimicrobial therapy
}

Yuchao Liang $\AA^{a}$, Hongjuan Zhang $\neq^{b}$, Huanxiang Yuan $*^{*}{ }^{b}$,Wen Lu ${ }^{b}$, Zelin Li ${ }^{b}$, Lei Wang*a, Lihua Gao*b

a Neurosurgery Department, Beijing Tian Tan Hospital, Capital Medical University, Beijing 100050, P. R. China

b Department of Chemistry, School of Science, Beijing Technology and Business University, Beijing 100048, P. R. China.

*E-mail: yhx@iccas.ac.cn,wanglei_tiantan@163.com,gaolh@th.btbu.edu.cn

\section{Experimental Section}

Materials and Measurements

DPPT-TT (200,000 Da) was purchased from Derthon Optoelectronic Materials Science Technology Co., Ltd. DPATP-CN was provided by Professor Cui-Hua Zhao. The Escherichia coli (E. coli) was purchased from Beijing Bio-Med Technology Development Co., Ltd. Candida albicans (C. albicans) ATCC 10231 and Staphylococcus aureus (S. aureus) were obtained from China General Microbiological Culture Collection Center. UV-Vis absorption spectra were taken on a JASCO V-550 
spectrophotometer. Fluorescence spectra were measured on a Hitachi F-4500 fluorometer equipped with a xenon lamp excitation source. Zeta potentials were measured on a Nano ZS (ZEN3600) system.

\section{Preparation of DDNPS}

$200 \mu \mathrm{L}$ of DPATP-CN solution $(1.0 \mathrm{mg} / \mathrm{mL}$, THF), $1 \mathrm{~mL}$ of DPPT-TT solution $(0.5$ $\mathrm{mg} / \mathrm{mL}$, THF), and $1 \mathrm{~mL}$ of PSMA solution ( $2 \mathrm{mg} / \mathrm{mL}$, THF) were mixed together and finally diluted to $4 \mathrm{~mL}$ of THF solution, then added into $10 \mathrm{~mL}$ of ultrapure water under ultrasound and was sonicated for 3-5 min to obtain a uniform transparent liquid. Next, nitrogen gas was purged to the finally obtained solution for $1 \mathrm{~h}$ to remove THF. Subsequently, the solution was concentrated to $5 \mathrm{~mL}$ under continuous nitrogen bubbling in a $100{ }^{\circ} \mathrm{C}$ oil bath. After cooling to room temperature, the solution was filtered through a $0.22 \mu \mathrm{m}$ filter to get the final DMCPNs dispersion.

The concentration of DMCPNs in aqueous solution was determined according to the following calculation. ${ }^{1} 4 \mathrm{~mL}$ of DMCPNs aqueous solution was freeze-dried to afford $2.8 \mathrm{mg}$ of powder. The density of DDNPs in aqueous solution is estimated as $1.1 \mathrm{~g}$. $\mathrm{mL}^{-1}$, and the average diameter of DDNPs is $70 \mathrm{~nm}$. The concentration of DDNPs could be calculated from the following equation.

Total number of DDNPs in $4 \mathrm{~mL}$ of solution $=\frac{\text { Total volume of DDNPS }}{\text { Average volume of each DDNPS }}$

$$
=\frac{\left(3.6 \times 10^{-3} \mathrm{~g}\right) /(1.1 \mathrm{~g} / \mathrm{mL})}{\frac{4}{3} \pi \times\left(35 \times 10^{-7}\right)^{3} \mathrm{~mL}}
$$


$=1.8 \times 10^{13}$

DDNPs concentration

$$
=\frac{\left(1.8 \times 10^{13}\right) /\left(6.02 \times 10^{23} \mathrm{~mol}\right)}{4 \times 10^{-3} \mathrm{~L}}=7.5 \times 10^{-3} \mu \mathrm{M}
$$

\section{Photothermal property characterization}

$100 \mu \mathrm{L}$ of DDNPs solution $\left(7.5 \times 10^{-3} \mu \mathrm{M}\right)$ was added in a $200 \mu \mathrm{L}$ tube and irradiated with an $808 \mathrm{~nm}$ laser for $5 \mathrm{~min}$ with a light intensity of $600 \mathrm{~mW} / \mathrm{cm}^{2}$. Temperature changes were detected and recorded using a thermal imager (accuracy of $\pm 0.1^{\circ} \mathrm{C}$ ).

\section{Antimicrobial experiment}

To $100 \mu \mathrm{L}$ of $C$. albicans suspension $\left(\mathrm{OD}_{600}=1.5\right)$ was added $10 \mu \mathrm{L}, 20 \mu \mathrm{L}$, and $30 \mu \mathrm{L}$ of DDNPs solution $\left(7.5 \times 10^{-3} \mu \mathrm{M}\right)$, and the final volume of the fungi solution was supplemented to $500 \mu \mathrm{L}$ with PBS. The above solution was placed in dark at $30{ }^{\circ} \mathrm{C}$ for 30 min and then a $100 \mu \mathrm{L}$ portion of each group was irradiated for 5 min under white light $\left(65 \mathrm{~mW} / \mathrm{cm}^{2}\right)$ followed by irradiation for $5 \mathrm{~min}$ by $808 \mathrm{~nm}$ laser $\left(600 \mathrm{~mW} / \mathrm{cm}^{2}\right)$. The $C$. albicans suspensions were diluted 500 fold with PBS. $100 \mu \mathrm{L}$ of the diluted fungi solution was applied on the YPD medium, and the number of colonies formed after incubation at $30{ }^{\circ} \mathrm{C}$ for 16 hours was counted. The irradiation conditions of the control group were protected from light, and the irradiation conditions of the experimental group were near-infrared (NIR) light irradiation, white light irradiation, and NIR + white light irradiation. The solid medium culture specification is $90 \mathrm{~mm}$. Calculate the $C$. albicans inhibition rate IR according to the following formula: 


$$
\mathrm{IR}=\frac{C_{0}-C}{C_{0}} \times 100 \%
$$

Where $\mathrm{C}$ is the number of colony forming units (cfu) of the experimental group, and $\mathrm{C}_{0}$ is the number of colony forming units of the control group.

For the case of $E$. coli and $S$. aureus, all the protocols are the same as above steps except that the suspensions of E. coli and S. aureus were diluted $1 \times 10^{4}$ fold before coating on the solid medium and the culture mediums are LB and NB for E. coli and S. aureus respectively.

\section{Photothermal conversion efficiency calculation}

Photothermal conversion efficiency $(\eta=70 \%)$ of DMCPNs is calculated according to the following equation:

$$
\eta=\frac{h S \Delta T_{\max }-Q_{D i s}}{I\left(1-10^{-A_{808}}\right)}
$$

where, $\mathrm{h}$ represents the heat transfer coefficient, $\mathrm{S}$ is the surface area of the vessel, $\Delta$ Tmax represents the maximum temperature variation, which is defined as Tmax-Tsurr, Tmax and Tsurr are the maximum steady-state temperature $\left(62.4{ }^{\circ} \mathrm{C}\right)$ and ambient temperature of the environment, respectively $\left(30{ }^{\circ} \mathrm{C}\right)$, Q $\mathrm{Q}_{\mathrm{Dis}}$ represents the heat associated with the light absorbance of the solvent and container, and the $\mathrm{Q}_{\text {Dis }}$ was evaluated independently to be $14 \mathrm{~mW}$ using pure water, I is laser power density $(0.6$ $\mathrm{W} \bullet \mathrm{cm}^{-2}$ ), and $\mathrm{A}_{808}(0.695)$ is the absorbance of DMCPNs at $808 \mathrm{~nm} \mathrm{hS}$ was calculated according to the following equation:

$$
\tau_{s}=\frac{m_{D} C_{D}}{h S}
$$


where, $\mathrm{m}_{\mathrm{D}}$ and $\mathrm{C}_{\mathrm{D}}$ are the mass $(0.5 \mathrm{~g})$ and heat capacity $(4.2 \mathrm{~J} / \mathrm{g})$ of pure water used as the solvent, respectively. To obtain $\tau_{\mathrm{s}}$, the other equation is used and as follows:

$\mathrm{t}=-\tau_{\mathrm{s}}(\operatorname{Ln} \theta)$

where $\theta$ is defined as the ratio of $\Delta \mathrm{T}$ and $\Delta \mathrm{Tmax}, \tau_{\mathrm{s}}$ is calculated to be $204.6 \mathrm{~s}$ from the Figure S2.

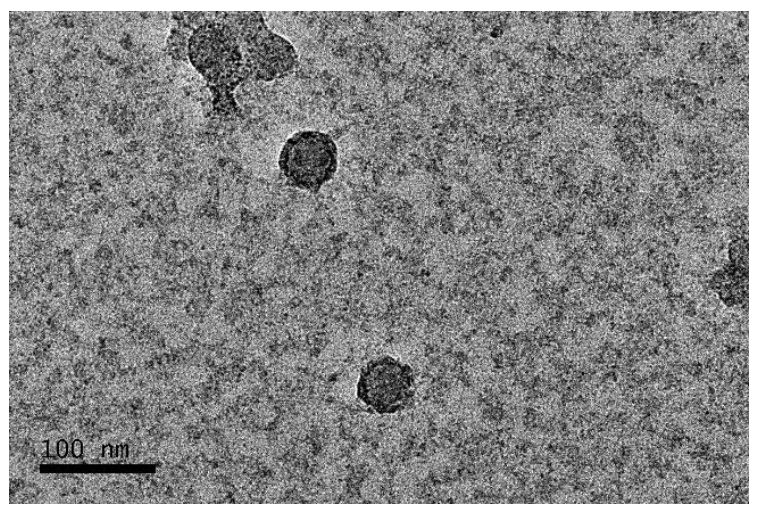

Figure S1 The TEM image of DDNPs

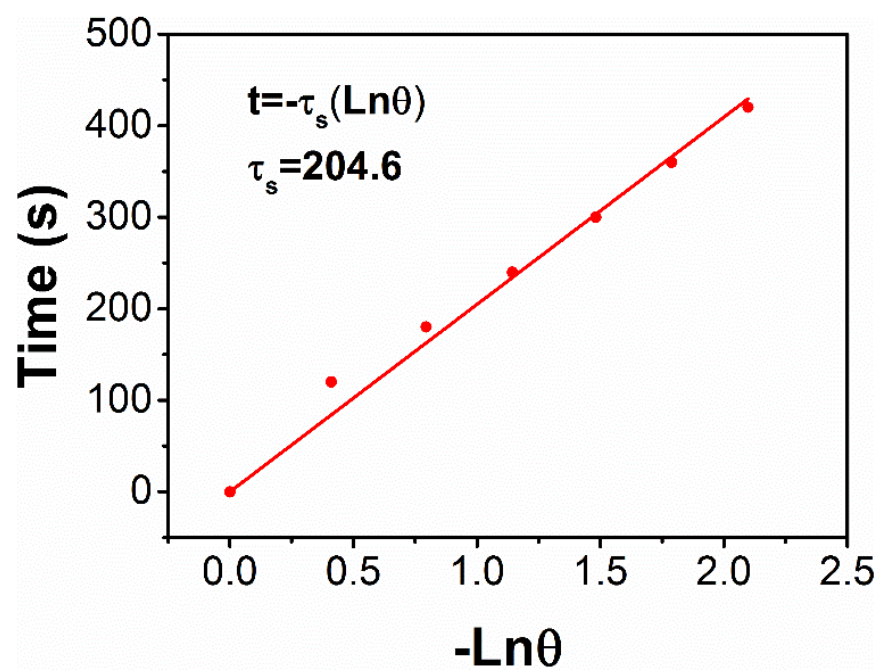

Figure S2 A plot of time against temperature during the cooling period in Figure 2c in the manuscript.

LUMO

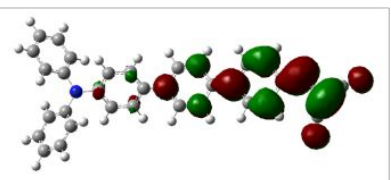

HOMO

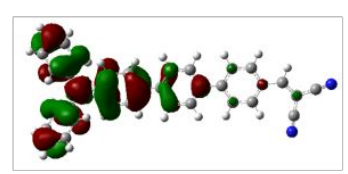

S-5
Calculated $\mathrm{T}_{1}$ energy level

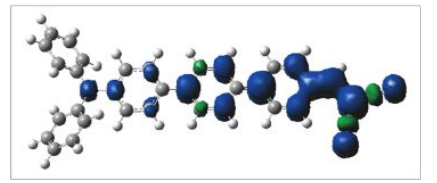


Figure S3 The calculated spatial distribution of lowest unoccupied molecular orbital (LUMO), highest occupied molecular orbital (HOMO), spin density plot of lowestlying triplet state $\left(\mathrm{T}_{1}\right)$ and $\mathrm{T} 1$ energy level.

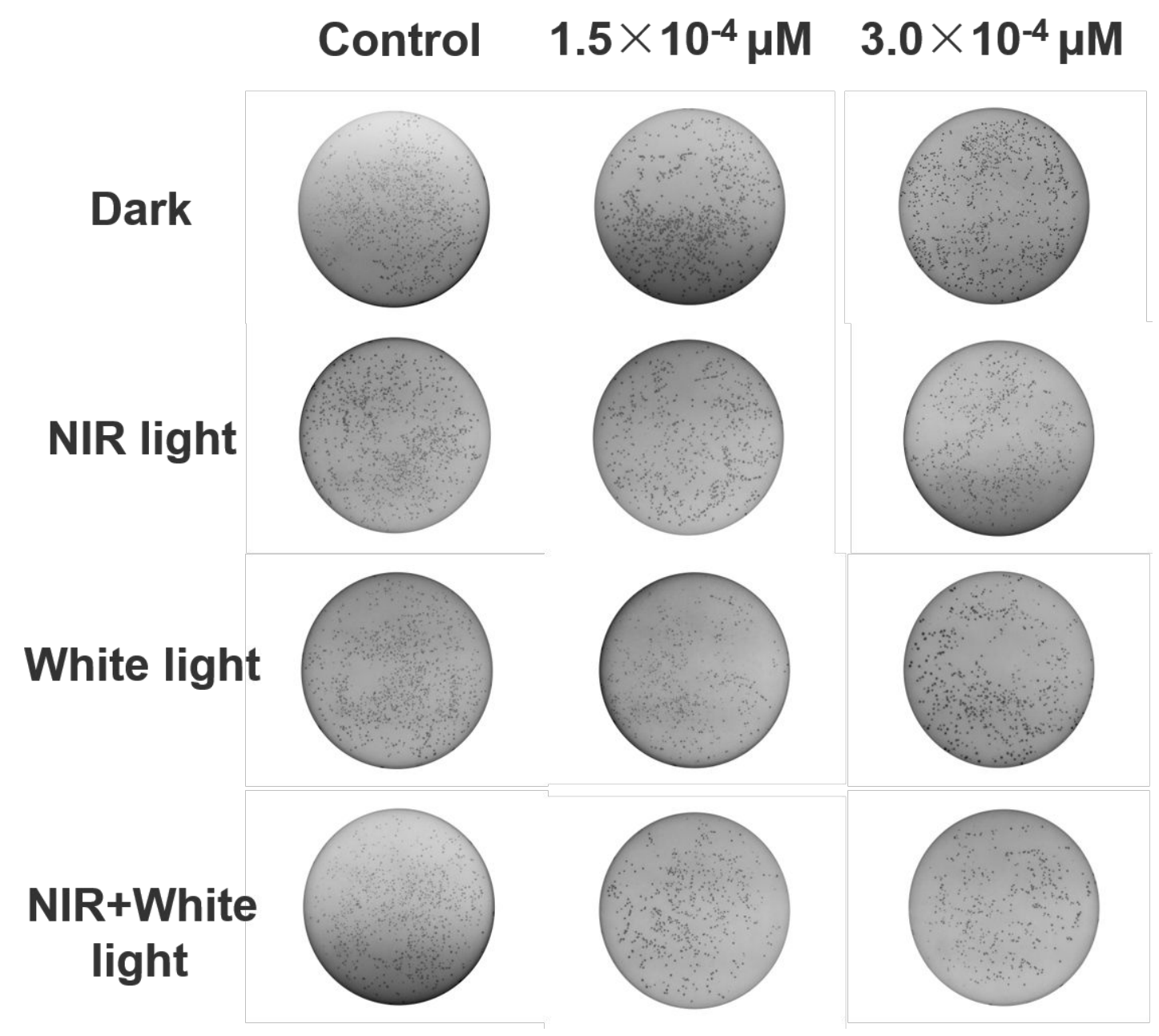

Figure S4 The plate photographs of $E$. coli interacted with $1.5 \times 10^{-4} \mu \mathrm{M}$ and $3.0 \times 10^{-4}$ $\mu \mathrm{M}$ of DDNPs in Dark, under NIR light, White light and NIR + White light. 


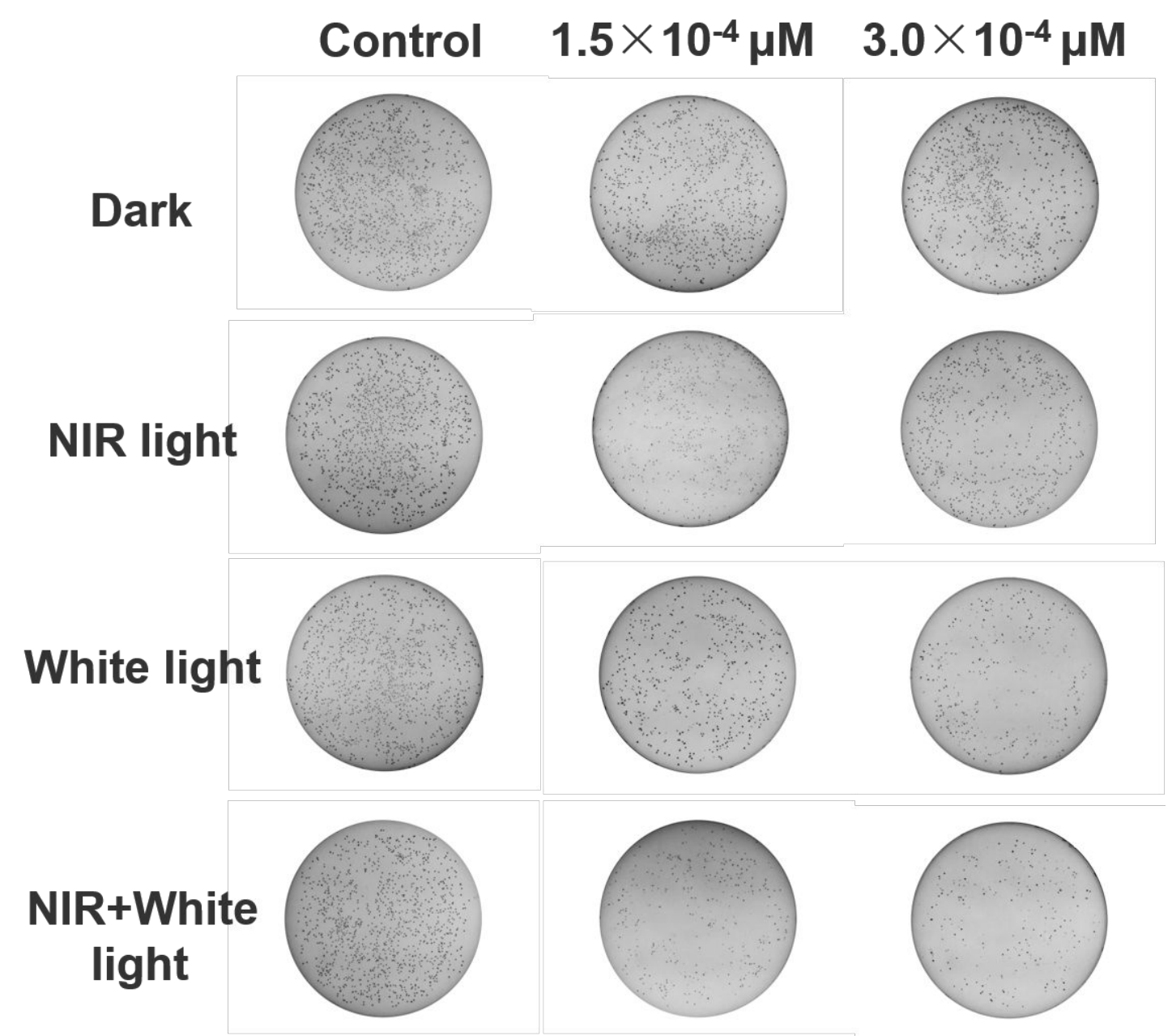

Figure S5 The plate photographs of $S$. aureus interacted with $1.5 \times 10^{-4} \mu \mathrm{M}$ and $3.0 \times 10^{-4}$ $\mu \mathrm{M}$ of DDNPs in Dark, under NIR light, White light and NIR + White light. 


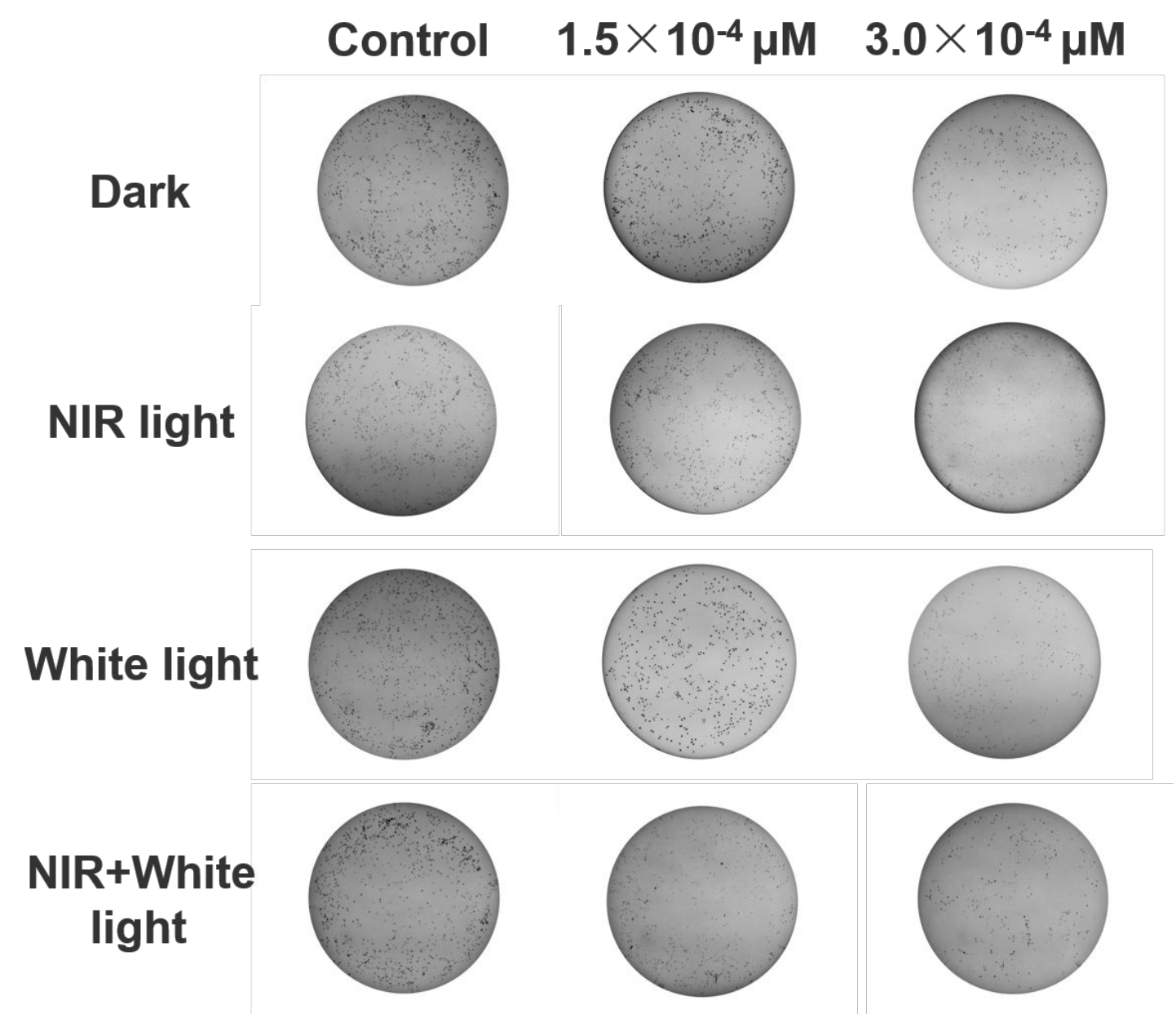

Figure S6 The plate photographs of $C$. albicans interacted with $1.5 \times 10^{-4} \mu \mathrm{M}$ and $3.0 \times 10^{-4} \mu \mathrm{M}$ of DDNPs in Dark, under NIR light, White light and NIR + White light. 


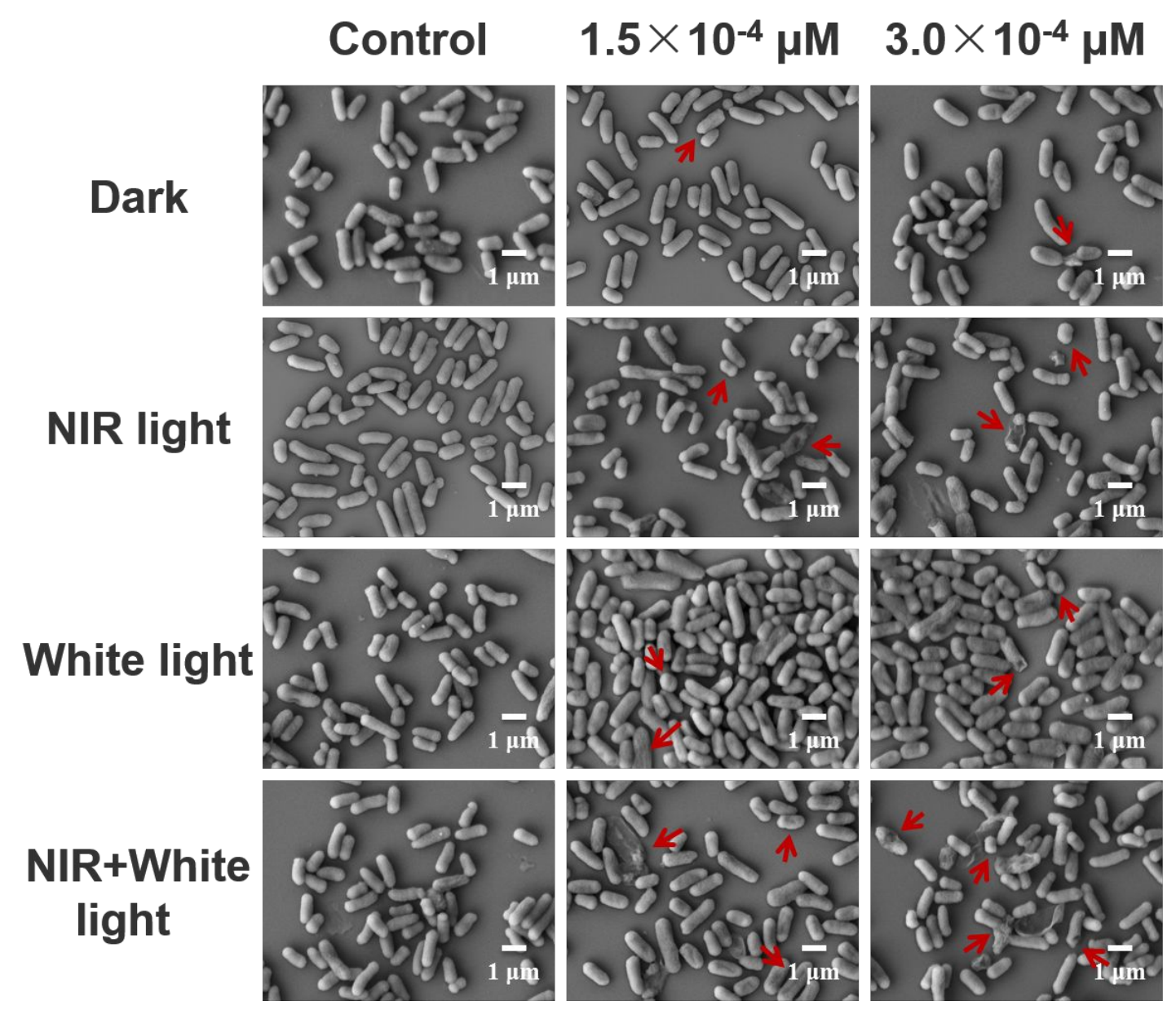

Figure S7 The SEM images of E. coli interacted with $1.5 \times 10^{-4} \mu \mathrm{M}$ and $3.0 \times 10^{-4} \mu \mathrm{M}$ of DDNPs in Dark, under NIR light, White light and NIR + White light. 


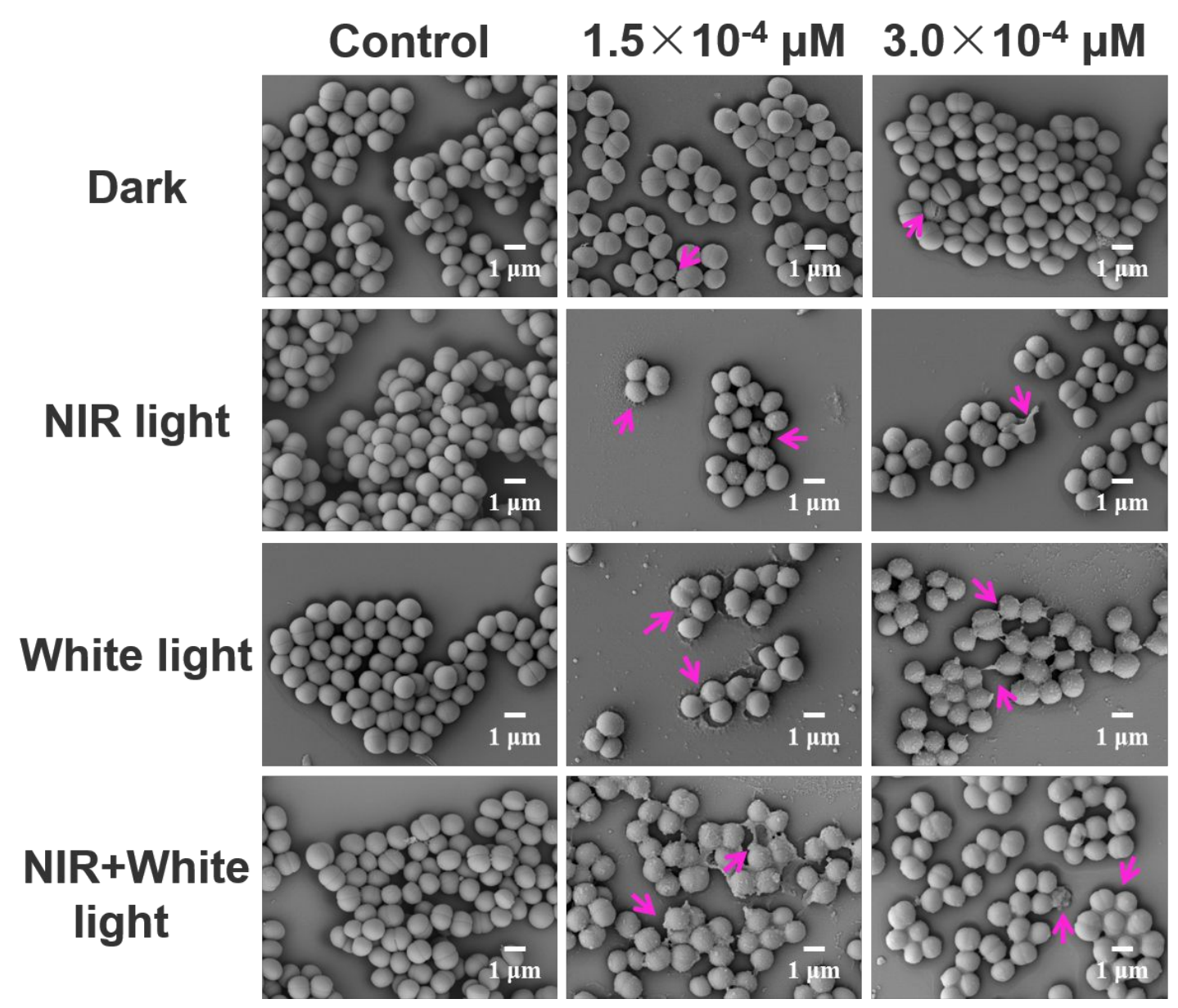

Figure S8 The SEM images of $S$. aureus interacted with $1.5 \times 10^{-4} \mu \mathrm{M}$ and $3.0 \times 10^{-4} \mu \mathrm{M}$ of DDNPs in Dark, under NIR light, White light and NIR + White light. 


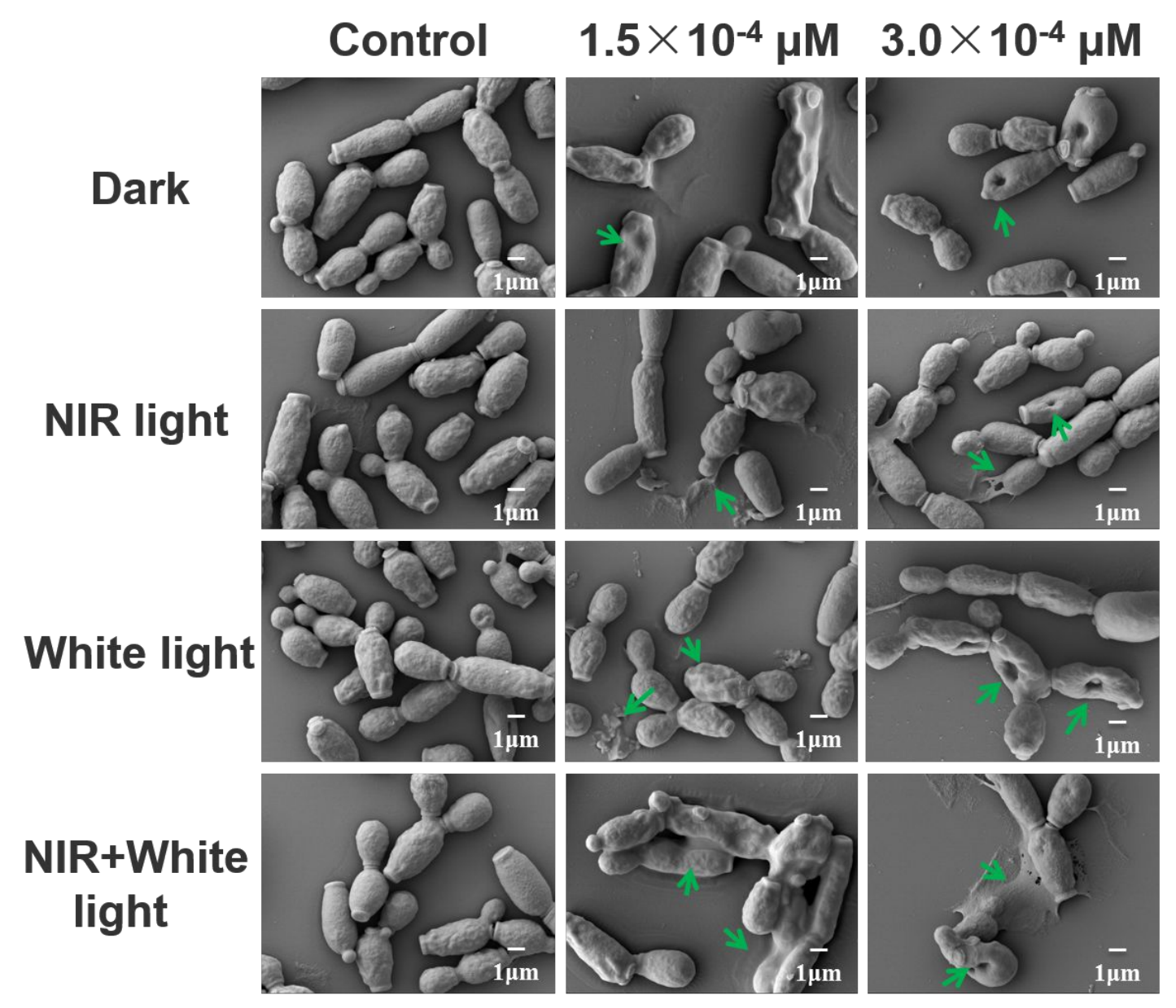

Figure S9 The SEM images of C. albicans interacted with $1.5 \times 10^{-4} \mu \mathrm{M}$ and $3.0 \times 10^{-4}$ $\mu \mathrm{M}$ of DDNPs in Dark, under NIR light, White light and NIR + White light.

The stability of nanoparticles is assessed in PBS and cell culture medium. The size of DDNPs in PBS solutions are nearly the same as that in aqueous solution, while the size of DDNPs in cell culture medium slightly increases because of the nonspecific adsorption to nanoparticle surface of proteins in cell medium. (Figure S10) 


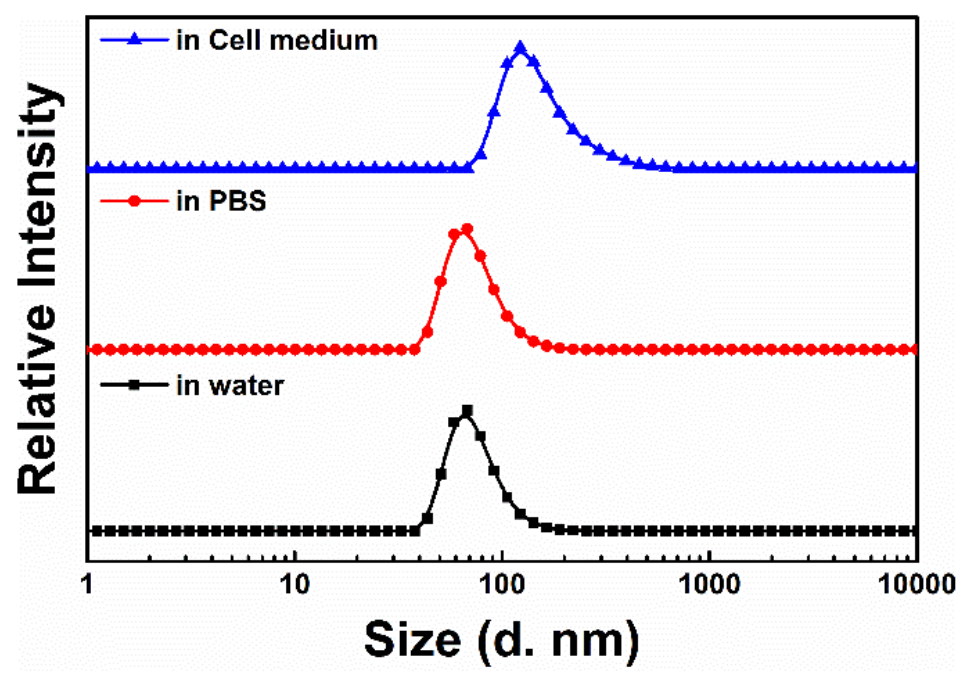

Figure S10 The sizes of DDNPs in water, PBS and cell medium.

The temperature change process of DDNPs aqueous solution under white light irradiation is monitored by a thermal infrared imager, and as seen from Figure S11, the results indicate white light cannot induce heat generation in the system.

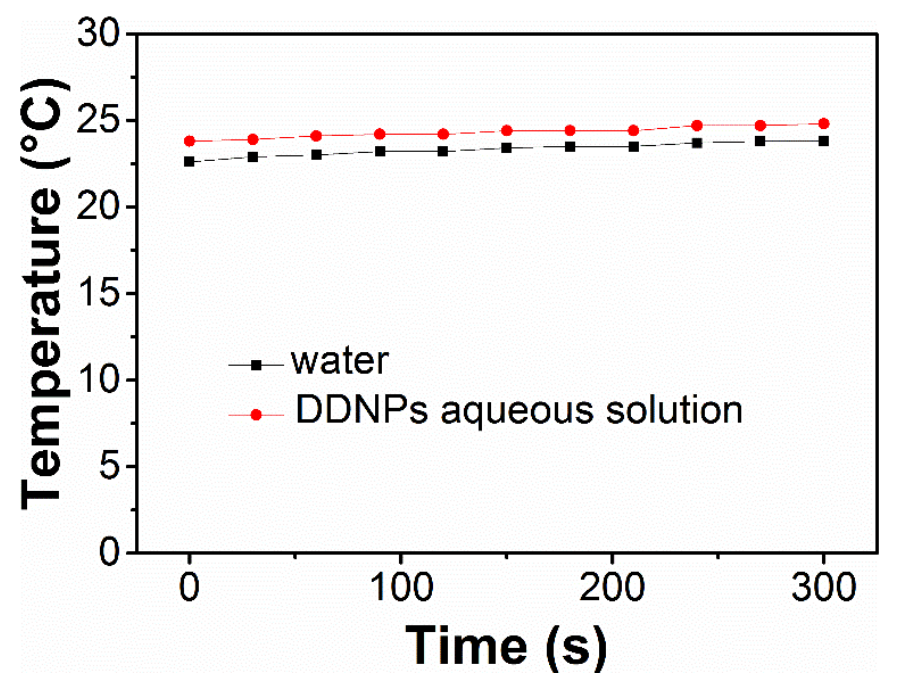

Figure S11 The temperature change of DDNPs aqueous solution under white light irradiation

The cytotoxicity of DDNPs is evaluated by a MTT method. DDNPs in relatively low concentration (lower than $4 \times 10^{-4} \mu \mathrm{M}$ ) exhibit good biocompatibility, as shown in Figure S12. 


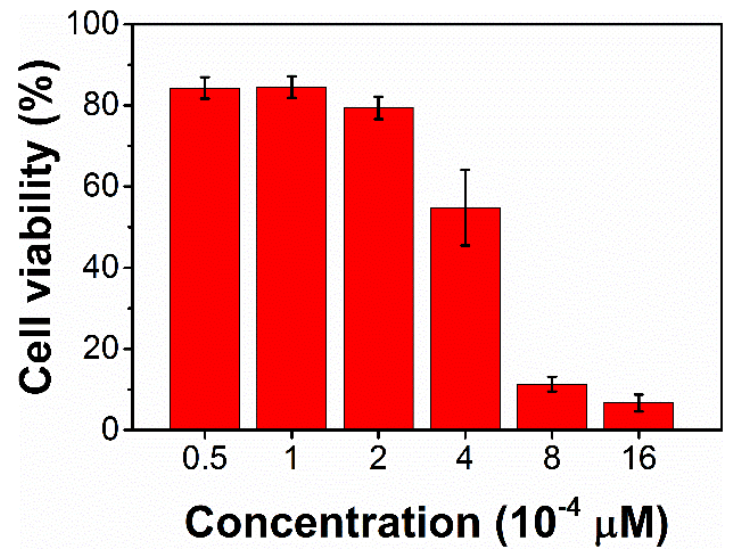

Figure S12 The cell viability of HeLa cells treated with $0.5 \sim 16 \times 10^{-4} \mu \mathrm{M}$ DDNPs.

Reference

(1) Feng, G.; Fang, Y.; Liu, J.; Geng, J.; Ding, D.; Liu, B. Multifunctional Conjugated Polymer Nanoparticles for Image-Guided Photodynamic and Photothermal Therapy. Small 2017, 13, 1602807 